\title{
New Integral Transform in Caputo Type Fractional Difference Operator
}

\author{
A. Fundo ${ }^{1}$, A. Kashuri ${ }^{2, *}$, R. Liko ${ }^{2}$ \\ ${ }^{1}$ Department of Mathematics, Polytechnic University of Tirana, Albania \\ ${ }^{2}$ Department of Mathematics, Faculty of Technical Science, University "Ismail Qemali", Albania \\ *Corresponding Author: artionkashuri@gmail.com
}

Copyright $(2016$ by authors, all rights reserved. Authors agree that this article remains permanently open access under the terms of the Creative Commons Attribution License 4.0 International License.

\begin{abstract}
In this paper, we introduce Caputo type nabla $(q, h)$-fractional difference operators and investigate their basic properties and also to show the applicability of this interesting $(q, h)$-new integral transform method and its efficiency in solving linear fractional difference equations. Differential equations with fractional derivative provide a natural framework for the discussion of various kinds of real problems modeled by the aid of fractional derivative. Discrete analogues of some topics of continuous fractional calculus have been developed. Finally, we provide the general solutions in terms of discrete Mittag-Leffler functions.
\end{abstract}

Keywords $(q, h)-$ New Integral Transform, Convolution, Fractional Difference Equations, Nabla $(q, h)$ - Fractional Integral, Nabla $(q, h)$ - Fractional Derivative, $(q, h)$-Calculus, Mittag-Leffler Functions

\section{Introduction}

$(q, h)$ - new integral transform is derived from the classical Fourier integral and was introduced by Artion Kashuri and Akli Fundo to facilitate the process of solving linear fractional difference equations in the time domain [6], [7]. $(q, h)$ - new integral transform is defined for functions of exponential order. Fractional calculus deals with the study of fractional order integrals and derivatives and their applications [1]. Riemann-Liouville and Caputo are kinds of fractional derivatives which generalize the ordinary integral and differential operators. Differential equations with fractional derivative provided a natural framework for the discussion of various kinds of real problems modeled by the aid of fractional derivative. Discrete analogues of some topics of continuous fractional calculus have been developed. The aim of this paper is to introduce Caputo type nabla $(q, h)$-fractional difference operators and investigate their basic properties, and also to show the applicability of this interesting $(q, h)-$ new integral transform method and its efficiency in solving linear fractional difference equations. Caputo type nabla $(q, h)$ - fractional difference operators is used in combination with $(q, h)$ - new integral transform as an application. By applying $(q, h)$ - new integral transform method we derive explicit solutions to the homogeneous equations of the form

${ }_{a}^{C} \nabla_{(q, h)}^{\alpha} \mathrm{y}(\mathrm{t})-\lambda \mathrm{y}(\mathrm{t})=0, d_{k}=\nabla^{k} y(a), k=0, m-1$. Here, we solve some linear fractional difference equations involving Caputo type $(q, h)$-derivatives and provide the general solutions in terms of discrete Mittag-Leffler functions.

\section{Preliminaries}

By a time scale $\mathbb{T}$ we understand any nonempty, closed subset of reals with the ordering inherited from reals. For any $t \in \mathbb{T}$, we define the forward and backward jump operators as $\sigma(\mathrm{t}):=\inf \{\mathrm{s} \in \mathbb{T}: \mathrm{s}>t\}$ and $\rho(\mathrm{t}):=$ $\sup \{\mathrm{s} \in \mathbb{T}: \mathrm{s}<t\}$, respectively. The forward and backward graininess functions are defined as $\mu(\mathrm{t}):=\sigma(\mathrm{t})-\mathrm{t}$ and $v(t):=t-\rho(t)$, respectively. We say that a point is left-dense if $\rho(\mathrm{t})=\mathrm{t}$ and left- scattered if $\rho(\mathrm{t}) \neq \mathrm{t}$. The right - dense and right-scattered points are defined in a similar manner. A point which is both left - and right - scattered is discrete. If $\inf \{\mathbb{T}\}=a_{0}>-\infty$, we define $\mathbb{T}_{\mathrm{k}}:=\mathbb{T} \backslash \mathrm{a}_{0}$, otherwise $\mathbb{T}_{\mathrm{k}}:=\mathbb{T}$. For a function $f: \mathbb{T} \rightarrow \mathbb{R}$ and a point $t \in \mathbb{T}_{k}$, we define $f^{\nabla}(t)$ to be a number such that for $\epsilon>0$ there exists a neighborhood $\mathcal{U} \subset \mathbb{T}$ of $\mathrm{t}$ which satisfies

$$
\begin{aligned}
& \left|(f(\rho(t))-f(\tau))-f^{\nabla}(t)[\tau-\rho(t)]\right| \leq \epsilon|\tau-\rho(t)| \text { for all } \\
& \tau \in \mathcal{U} \text {. }
\end{aligned}
$$

If $f^{\nabla}(t)$ is defined for all $t \in \mathbb{T}_{k}$, then the function obtained is called the $\nabla$-derivative of $\mathrm{f}$. A function $\mathrm{f}: \mathbb{T} \rightarrow \mathbb{R}$ is called left-dense continuous or ld-continuous provided it is continuous at every left-dense point in $\mathbb{T}$, and $f(t+)$ exists for every right-dense point in $\mathbb{T}$, and the set of $1 d$-continuous functions is denoted by $\left(C_{l d}(\mathbb{T})\right)$. If 
$f \in\left(C_{l d}(\mathbb{T})\right)$, then there is a function $F$ such that $F^{\nabla}(t)=f(t)$. In this case, we define the $\nabla$-integral as

$$
\int_{\mathbf{a}}^{t} \mathbf{f}(\boldsymbol{\tau}) \nabla \boldsymbol{\tau}=\mathbf{F}(\mathbf{t})-\mathbf{F}(\mathbf{a}) \text { for all } \mathbf{t} \in \mathbb{T} .
$$

Clearly, if $\mathbf{f}: \mathbb{T} \rightarrow \mathbb{R}$ is ld-continuous and $\mathbf{t} \in \mathbb{T}_{\mathbf{k}}$, then

$$
\int_{\rho(t)}^{t} \mathbf{f}(\tau) \nabla \tau=v(t) \mathbf{f}(\mathbf{t}) \text {. }
$$

\section{Definition 2.1.}

A function $f \in\left(C_{l d}(\mathbb{T})\right)$ is called $v$-regressive if $1+\mathrm{fv} \neq 0$ on $\mathbb{T}_{\mathrm{k}}$, and $\mathrm{f} \in\left(\mathrm{C}_{\mathrm{ld}}(\mathbb{T})\right)$ is called positively-regressive if $1+f v>0$ on $\mathbb{T}_{k}$.

The set of $v$-regressive functions and the set of positively $v-$ regressive functions are denoted by $\left(\mathcal{R}_{v}(\mathbb{T})\right)$ and $\left(\mathcal{R}_{v}^{+}(\mathbb{T})\right)$, respectively. For simplicity, we denote by $\left({ }_{c} \mathcal{R}_{v}(\mathbb{T})\right)$ the set of $v$-regressive constants. Let $\lambda \in\left({ }_{c} \mathcal{R}_{v}(\mathbb{T})\right)$ and $s \in \mathbb{T}$, then the generalized exponential function $\hat{\mathrm{e}}_{\lambda}(\cdot, \mathrm{s})$ on time scale $\mathbb{T}$ is denoted by the unique solution of the initial value problem

$$
\left\{\begin{array}{l}
x^{\nabla}(t)=\lambda x(t), \quad t \in \mathbb{T}_{k} \\
x(s)=1
\end{array}\right.
$$

For $\mathrm{p} \in\left({ }_{c} \mathcal{R}_{v}(\mathbb{T})\right)$, define circle minus $\mathrm{p}$ by

$$
\ominus_{v} \mathrm{p}:=-\frac{\mathrm{p}}{1-\mathrm{pv}} \text {. }
$$

Then the unique solution of the initial value problem

$$
\left\{\begin{array}{l}
x^{\nabla}(t)=-\lambda x(t), \quad t \in \mathbb{T}_{k} \\
x(s)=1
\end{array}\right.
$$

takes the form $\hat{\mathrm{e}}_{\Theta_{v} \lambda}(\cdot, \mathrm{s})$. It is known that the exponential function $\hat{\mathrm{e}}_{\mathrm{f}}(\cdot, \mathrm{s})$ is strictly positive on $[\mathrm{s}, \infty[\mathbb{T}$ provided that $\mathrm{f} \in \mathcal{R}^{+}\left(\left[\mathrm{s}, \infty\left[{ }_{\mathbb{T}}\right)\right.\right.$. The definition of the generalized monomials $\hat{\mathrm{h}}_{\mathrm{n}}: \mathbb{T} \times \mathbb{T} \rightarrow \mathbb{R}\left(\mathrm{n} \in \mathbb{N}_{0}\right)$ is given by

$$
\hat{\mathrm{h}}_{\mathrm{n}}(\mathrm{t}, \mathrm{s})= \begin{cases}1, & \mathrm{n}=0 \\ \int_{\mathrm{s}}^{\mathrm{t}} \hat{\mathrm{h}}_{\mathrm{n}-1}(\tau, \mathrm{s}) \nabla \tau, & \mathrm{n} \in \mathbb{N} .\end{cases}
$$

for $s, t \in \mathbb{T}$, [2]. If we let $\hat{h}_{n}^{\nabla}(t, s)$ denote for each fixed $s \in \mathbb{T}$ the derivatives of $\hat{h}_{n}^{\nabla}(t, s)$ with respect to $t$, then

$$
\hat{\mathrm{h}}_{\mathrm{n}}^{\nabla}(\mathrm{t}, \mathrm{s})=\hat{\mathrm{h}}_{\mathrm{n}-1}(\mathrm{t}, \mathrm{s}) \text { for } \mathrm{n} \in \mathbb{N}, \mathrm{t} \in \mathbb{T}_{\mathrm{k}} \text {. }
$$

An important relation between the generalized exponential function and the monomials is given by

$$
\hat{\mathrm{e}}_{\lambda}(\mathrm{t}, \mathrm{s})=\sum_{\mathrm{k}=0}^{\infty} \lambda^{\mathrm{k}} \hat{\mathrm{h}}_{\mathrm{k}}(\mathrm{t}, \mathrm{s}) \text { for } \mathrm{s}, \mathrm{t} \in \mathbb{T} \text { with } \mathrm{t} \geq \mathrm{s},
$$

where $\lambda \in\left(\mathcal{R}_{\mathrm{c}}(\mathbb{T})\right)$.

\section{Definition 2.2.}

The nabla new integral transform ([6]-[8]) of a function $\mathrm{f}: \mathbb{T} \rightarrow \mathbb{R}$ is defined by

$\widehat{K}_{\nabla}\{f\}(z, v):=\frac{1}{v} \int_{v}^{\infty} \hat{\mathrm{e}}_{\ominus v_{v} \frac{1}{v^{2}}}(\rho(\tau), \mathrm{z}) f(\tau) \nabla \tau$ for $z \in D$,

where $D$ consists of all complex numbers $z \in \mathbb{C}$ for which the improper $\nabla$-integral exists.

Definition 2.3.
The convolution of two functions $f, g: \mathbb{T} \rightarrow \mathbb{R}$ is defined by

$$
(f * g)(t):=\int_{a}^{t} \hat{f}(t, \rho(s)) \mathrm{g}(\mathrm{s}) \nabla \mathrm{s}, \quad \mathrm{t} \in \mathbb{T},
$$

where $\hat{f}$ is the shift of $f$ introduced in [3]. Then

$$
\widehat{K}_{\nabla}\{f * g\}(z, v)=z \cdot \widehat{K}_{\nabla}\{f\}(z, v) \cdot \widehat{K}_{\nabla}\{g\}(z, v) .
$$

The following result is the nabla version of the result obtained in [4] (change of integration order). Let $f,: \mathbb{T} \rightarrow \mathbb{R}$ and $f \in\left(C_{l d}\left(\mathbb{T}^{2}\right)\right)$, then

$$
\int_{s}^{t} \int_{s}^{\eta} f(\eta, \zeta) \nabla \zeta \nabla \eta=\int_{s}^{t} \int_{\rho(\zeta)}^{t} f(\eta, \zeta) \nabla \eta \nabla \zeta \quad \text { for } s, \mathrm{t} \in \mathbb{T} .
$$

\section{3. $(q, h)$-Fractional Calculus}

Consider the following $(q, h)$-time scale:

$$
\mathbb{T}_{(q, h)}^{t_{0}}=\left\{t_{0} q^{k}+[k]_{q} h: k \in \mathbb{Z}\right\} \cup\left\{\frac{h}{1-q}\right\},
$$

for $t_{0}>0, q \geq 1, h \geq 0$ and $q+h>1$. Note that if $q=1$, then the cluster point $h / 1-q=-\infty$ is not involved in $\mathbb{T}$. The forward and backward jump operator is the linear function $\sigma(\mathrm{t})=\mathrm{qt}+\mathrm{h}$ and $\rho(\mathrm{t})=q^{-1}(t-h)$, respectively. Similarly, the forward and backward graininess is given by $\mu(\mathrm{t})=(\mathrm{q}-1) \mathrm{t}+\mathrm{h}$ and $v(\mathrm{t})=$ $q^{-1} \mu(t)$, respectively. Observe that

$\sigma^{k}(t)=q^{k} t+[k]_{q} h$ and $\rho^{k}(t)=q^{-k}\left(t-[k]_{q} h\right)$.

The following relation $v\left(\rho^{k}(t)\right)=q^{-k} v(\mathrm{t})$ holds for $t \in \mathbb{T}$.

\section{Definition 3.1.}

The nabla $(q, h)$-derivative of the function $\mathrm{f}: \mathbb{T}_{(q, h)}^{t_{0}} \rightarrow$ $\mathbb{R}$ is defined by

$$
\nabla_{(q, h)} f(t):=\frac{f(t)-f(\rho(t))}{v(\mathrm{t})}=\frac{f(t)-f(\tilde{q}(t-h))}{(1-\tilde{q}) t+\tilde{q} h},
$$

where $\tilde{q}=q^{-1}$. Let $\mathrm{t}, \mathrm{a} \in \mathbb{T}_{(q, h)}^{t_{0}}$ such that $h / 1-q \leq$ $a \leq t$ and $\mathrm{f}: \mathbb{T} \rightarrow \mathbb{R}$. Then the nabla $(q, h)-$ integral exists and can be calculated (provided $t>a$ ) via the formula

$$
\begin{array}{r}
{ }_{a} \nabla_{(q, h)}^{-1} f(t):= \\
\int_{a}^{t} f(\tau) \nabla \tau=\left(\left(1-q^{-1}\right) t+q^{-1} h\right) \sum_{k=0}^{n-1} q^{-k} f\left(q^{-k} t+\right. \\
\left.[-k]_{q} h\right)
\end{array}
$$

\section{Definition 3.2.}

The Taylor monomials and the power functions on $\mathbb{T}_{(q, h)}^{t_{0}}$ have the forms

$$
\hat{h}_{n}(t, s)=\frac{\prod_{j=0}^{n-1}\left(\sigma^{j}(t)-s\right)}{[n]_{q} !}=\frac{\prod_{j=0}^{n-1}\left(t-\rho^{j}(s)\right)}{[n]_{q} !}
$$

where $(t-s)_{(\tilde{q}, h)}^{(n)}=\prod_{j=0}^{n-1}\left(t-\rho^{j}(s)\right)$. 
Respectively, and the extension of the monomials $\hat{h}_{n}(t, s)$ corresponding to $\mathbb{T}_{(q, h)}^{t_{0}}$ takes the form

$$
\hat{h}_{\alpha}(t, s)=\frac{(t-s)_{(\tilde{q}, h)}^{(\alpha)}}{\Gamma_{\tilde{q}}^{(\alpha+1)}}, \quad \alpha \in \mathbb{R} .
$$

\section{Lemma 3.3.}

[5] Let $m \in \mathbb{Z}^{+}, \alpha \in \mathbb{R}, \mathrm{s}, \mathrm{t} \in \mathbb{T}_{(q, h)}^{t_{0}} \quad$ and $n \in \mathbb{Z}^{+}$, $n \geq m$ be such that $t=\sigma^{n}(s)$. Then

$$
\nabla_{(q, h)}^{m} \hat{h}_{\alpha}(t, s)= \begin{cases}\hat{h}_{\alpha-m}(t, s), & \alpha \notin\{0,1, \ldots, m-1\}, \\ 0, & \alpha \in\{0,1, \ldots, m-1\} .\end{cases}
$$

Let $\alpha \in \mathbb{T}_{(q, h)}^{t_{0}}, a>h /(1-q)$ be fixed. We consider the following restricted $(q, h)$-time scale:

$$
\widetilde{\mathbb{T}}_{(q, h)}^{\sigma^{i}(a)}=\left\{t \in \mathbb{T}, \mathrm{t} \geq \sigma^{i}(a)\right\}, i=0,1,2, \ldots,
$$

where the symbol $\sigma^{i}$ stands for the $i$ th iterate of $\sigma$ (analogously, we use the symbol $\rho^{i}$ ). Now we can continue with the introduction of $(q, h)$-fractional integral and $(q, h)$ - fractional derivative of a function $\mathrm{f}: \widetilde{\mathbb{T}}_{(q, h)}^{\sigma^{i}(a)} \rightarrow \mathbb{R}$. Let $t \in \widetilde{\mathbb{T}}_{(q, h)}^{\sigma^{i}(a)}$.

\section{Definition 3.4.}

5] The nabla $(q, h)$-fractional integral (in the sense of Riemann-Liouville) of order $\alpha \in \mathbb{R}^{+}$over the time scale interval $[a, t] \cap \widetilde{\mathbb{T}}_{(q, h)}^{a}$ is defined by

$$
{ }_{a} \nabla_{(q, h)}^{-\alpha} f(t)=\int_{\mathrm{a}}^{\mathrm{t}} \hat{\mathrm{h}}_{\alpha-1}(\mathrm{t}, \rho(\tau)) \mathrm{f}(\tau) \nabla \tau
$$

\section{Definition 3.5.}

The nabla $(q, h)$-fractional derivative (in the sense of Caputo) of order $\alpha \in \mathbb{R}^{+}$is defined by

$$
\begin{gathered}
{ }_{a}^{C} \nabla_{(q, h)}^{\alpha} f(t)={ }_{a} \nabla^{-(m-\alpha)} \nabla^{m} f(t)= \\
=\int_{\mathrm{a}}^{\mathrm{t}} \hat{\mathrm{h}}_{\mathrm{m}-\alpha-1}(\mathrm{t}, \rho(\tau)) \nabla^{m} \mathrm{f}(\tau) \nabla \tau,
\end{gathered}
$$

where $m \in \mathbb{Z}^{+}$is such that $m-1<\alpha \leq m$.

\section{Application}

In this section we give solutions for fractional initial value problems on $a \in \mathbb{T}_{(q, h)}^{t_{0}}$.

\section{Definition 4.1.}

Let $\alpha, \beta, \gamma \in \mathbb{R}$. The $(q, h)-$ Mittag-Leffler function $E_{\alpha, \beta}^{s, \lambda}(t)$ is defined by

$$
\begin{array}{r}
E_{\alpha, \beta}^{s, \lambda}(t)= \\
\sum_{k=0}^{\infty} \lambda^{k} \hat{h}_{\alpha k+\beta-1}(t, s) \quad\left(=\sum_{k=0}^{\infty} \lambda^{k} \frac{{ }^{(t-s)}{ }_{(\tilde{q}, h)}^{(\alpha k+\beta-1)}}{\Gamma_{\tilde{q}}(\alpha k+\beta)}\right), \quad \text { (22) }
\end{array}
$$

for $s, t \in \widetilde{\mathbb{T}}_{(q, h)}^{\sigma(a)}$ and $t \geq s$. It is easy to check that the series on the right-hand side converges (absolutely) if $|\lambda|(v(\mathrm{t}))^{\alpha}<1$. Now we give $(q, h)-$ new integral transforms of fractional nabla integral and Caputo nabla derivative on $\mathbb{T}_{(q, h)}^{t_{0}}$. For $t, s \in \mathbb{T}_{(q, h)}^{t_{0}}$ and $\alpha \in \mathbb{R}^{+}$, we have

$$
\widehat{K}_{(q, h)}\left\{\hat{h}_{\alpha}(t, v)\right\}(z)=z^{2 \alpha+1} .
$$

From the definition of $(q, h)$-Mittag-Leffler function $E_{\alpha, \beta}^{s, \lambda}(t)$, we have

$$
\begin{gathered}
\widehat{K}_{(q, h)}\left\{E_{\alpha, \beta}^{a, \lambda}(t)\right\}(z)=\widehat{K}_{(q, h)}\left\{\sum_{k=0}^{\infty} \lambda^{k} \hat{h}_{\alpha k+\beta-1}(\cdot, a)\right\}(z) \\
=\sum_{k=0}^{\infty} \lambda^{k} \widehat{K}_{(q, h)}\left\{\hat{h}_{\alpha k+\beta-1}(\cdot, a)\right\}(z)=\frac{z^{2 \beta-1}}{1-\lambda z^{2 \alpha}} \\
\text { provided }\left|z^{2 \alpha} \lambda\right|<1 .
\end{gathered}
$$

For $\alpha=1, \beta=1$ and $t \in \mathbb{T}_{(q, h)}^{\sigma^{i}(a)}$, we have

$$
E_{1,1}^{a, \lambda}(t)=\hat{e}_{\lambda}(t, a) \text {. }
$$

Hence

$$
\widehat{K}_{(q, h)}\left\{\hat{e}_{\lambda}(t, a)\right\}(z)=\widehat{K}_{(q, h)}\left\{E_{1,1}^{a, \lambda}(t)\right\}(z)=\frac{z}{1-\lambda z^{2}} .
$$

\section{Theorem 4.2.}

The $(q, h)$-new integral transform of fractional nabla integral is given by

$$
\widehat{K}_{(q, h)}\left\{a_{(q, h)}^{-\alpha} f\right\}(z)=z^{2 \alpha} \cdot \widehat{K}_{(q, h)}\{f\}(z) .
$$

Proof. By convolution, one may write

$$
\begin{aligned}
{ }_{a} \nabla_{(q, h)}^{-\alpha} f(t) & =\int_{\mathrm{a}}^{\mathrm{t}} \hat{\mathrm{h}}_{\alpha-1}(\mathrm{t}, \rho(\tau)) f(\tau) \nabla \tau= \\
= & \left(\hat{\mathrm{h}}_{\alpha-1}(\cdot, a) * f\right)(t) .
\end{aligned}
$$

Thus

$$
\begin{gathered}
\widehat{K}_{(q, h)}\left\{{ }_{a} \nabla_{(q, h)}^{-\alpha} f\right\}(z)=z \cdot \widehat{K}_{(q, h)}\left\{\hat{h}_{\alpha-1}(t, a)\right\}(z) \cdot \widehat{K}_{(q, h)}\{f\}(z) \\
=z^{2 \alpha} \cdot \widehat{K}_{(q, h)}\{f\}(z) .
\end{gathered}
$$

\section{Theorem 4.3.}

The $(q, h)$ - new integral transform of Caputo nabla derivative is given by

$$
\widehat{K}_{(q, h)}\left\{{ }_{a}^{C} \nabla_{(q, h)}^{\alpha} f\right\}(z)=\frac{\widehat{K}_{(q, h)}\{f\}(z)}{z^{2 \alpha}}-\sum_{k=0}^{m-1} \frac{\nabla_{(q, h)}^{k} f(a)}{z^{2(\alpha-k)-1}},
$$

where $m-1<\alpha \leq m$. In particular, if $0<\alpha \leq 1$, then

$$
\widehat{K}_{(q, h)}\left\{{ }_{a}^{C} \nabla_{(q, h)}^{\alpha} \mathrm{f}\right\}(z)=\frac{\widehat{K}_{(q, h)}\{f\}(z)}{z^{2 \alpha}}-\frac{f(a)}{z^{2 \alpha-1}},
$$

where $\nabla_{(q, h)}^{0} f(a)=f(a), k=0 \quad$ and $m=1$. As an application, we apply $(q, h)$ - new integral transform method to derive explicit solutions to the homogeneous equations of the form 


$$
\begin{gathered}
{ }_{a}^{C} \nabla_{(q, h)}^{\alpha} \mathrm{y}(\mathrm{t})-\lambda \mathrm{y}(\mathrm{t})=0, \quad d_{k}=\nabla^{k} y(a), \\
k=0, \ldots, m-1,
\end{gathered}
$$

where $t \in \widetilde{\mathbb{T}}_{(q, h)}^{\sigma^{m}(a)}(m \in \mathbb{N}), m-1<\alpha \leq m, \lambda \in \mathbb{R}$, in terms of the $(q, h)-$ Mittag-Leffler functions. The following statement holds.

\section{Theorem 4.4.}

Let $m \in \mathbb{N}$ be given by $m-1<\alpha \leq m$ and $\lambda \in \mathbb{R}$. Then the functions

$$
y_{i}(t)=E_{\alpha, i+1}^{a, \lambda}(t), \quad i=0,1, \ldots, m-1,
$$

yield the fundamental system of solutions to equation (30).

Proof. Applying $(q, h)-$ new integral transform to (30) and taking (28) into account, we have

$$
\widehat{K}_{(q, h)}\{y\}(z)=\sum_{i=0}^{m-1} d_{i} \frac{z^{2 i+1}}{1-\lambda z^{2 \alpha}} .
$$

Formula (23) with $\beta=i+1$ yields

$$
\widehat{K}_{(q, h)}\left\{E_{\alpha, i+1}^{a, \lambda}(t)\right\}(z)=\frac{z^{2 i+1}}{1-\lambda z^{2 \alpha}} .
$$

Thus, relation (32) yields

$$
y(t)=\sum_{i=0}^{m-1} d_{i} y_{i}(t) \text {, where } y_{i}(t)=E_{\alpha, i+1}^{a, \lambda}(t) .
$$

\section{Conclusions}

In this paper, we introduce Caputo type nabla $(q, h)$-fractional difference operators and investigate their basic properties. Also we show the applicability of this interesting $(q, h)$-new integral transform method and its efficiency in solving linear fractional difference equations.
Finally, we provide the general solutions in terms of discrete Mittag-Leffler functions.

\section{Acknowledgements}

Express our sincere gratitude to Associate Professor Akli Fundo, whose suggestion led to conduction of this research.

\section{REFERENCES}

[1] M.R.S. Rahmat, M.S.M. Noorani: Caputo type fractional difference operator and its application on discrete time scales, Advanced in Difference Equations, 160, (2015).

[2] B.M. Peterson, A (eds.): Advanced in Dynamic Equations on Time Scales, Birkhäuser, Boston, (2003).

[3] Kisela, T: Power functions and essentials of fractional calculus on isolated time scales, Adv. Differ. Equ., 259, (2013).

[4] Karpuz: Volterra theory on time scales, Results Math., 65, 263-292, (2014).

[5] Cermak, J, Kisela, T, Nechvatal, L: Discrete Mittag-Leffler functions in linear fractional Difference equations, Abstr. Appl. Anal., (2011).

[6] A. Kashuri, A. Fundo: A New Integral Transform, Advanced in Theoretical and Applied Mathematics, 8, 1, 27-43, (2013).

[7] A. Kashuri, A. Fundo, M. Kreku: Mixture of a New Integral Transform and Homotopy Perturbation Method for Solving Nonlinear Partial Differential Equations, Advances in Pure Mathematics, 3, 3, 317-323, (2013).

[8] A. Kashuri, A. Fundo, R. Liko: New Integral Transform for Solving Some Fractional Differential Equations, International Journal of Pure and Applied Mathematics, 103, 4, 675-682, (2015). 